\title{
Study on Influencing Factors of Children's Behavior in Rural China
}

\author{
Haojin Wang \\ School of Politics and Administration \\ Wuhan University of Technology \\ Wuhan,P.R. China \\ Email: wanghaojin1995@163.com
}

\author{
Yanping $\mathrm{Yu}$ \\ School of Politics and Administration \\ Wuhan University of Technology \\ Wuhan,P.R. China \\ Email: yanpingyu@whut.edu.cn
}

\begin{abstract}
The research of rural children's behavior status mostly focuses on rural left-behind children, but there is few researches on the behavior of rural children as a whole. In order to understand the behavior status of rural children and the influencing factors of children's behavior in China, this paper conducts a survey among 4,889 rural children in Central China with new variables. By using factor analysis method, the results indicate: (a) Rural children does not only have negative behaviors, but also they perform in positive ways. (b) The three factor model is the best explanation of the data. The behaviors of seeking help from organizations, communication and learning as well as seeking help from individuals are successively important factors influencing the behavior status of rural children. It is suggested that social organizations should play an active role in serving rural children, families and the government should strengthen parenting education in rural areas, and that schools should improve the quality of teachers in rural areas so that the rural children can seek help from organizations and professionals when encountering difficulties. Joint efforts should be made to enhance the rural children's learning and communication capacity.
\end{abstract}

Keywords-rural children, behavior status, factor analysis; China

\section{INTRODUCTION}

The survival, protection and development of children are the basis of improving the quality of population, which is related to the future and destiny of the nation. Because of unbalanced development of the rural an urban areas, Chinese children in rural areas are faced with poor living environment, poor implementation of education policy and low educational level of their parents. They are a group of children needing more care and help. How to promote sound development of their behavior from the perspective of their behavior status and influencing factors is worthy of attention.

\section{LITERATURE REVIEW}

At present, the research on the behavior of rural children in China is as follows: With regard to the behavior of learning, academic circles have different findings on whether parents' long-term absence due to their working in cities has a greater negative impact on children's learning. Tang's study in 2011 shows that separation from parents has a significant negative impact on the weariness and learning achievement of left-behind children [1]. Ji's research (2016) found that there is

Fund support: Project sponsored by National College Students' Innovation and Entrepreneurship Training Program in 2017 (Project number: 20171049719005) no notable correlation between the learning attitude, learning achievement and the fact whether the children are left behind or not, but it shows that left-behind children have no clear learning objectives and they have low requirements for their own study [2]. With regard to life, Li discovered through his survey of 2,178 junior high school students in 2012 that parents as migrant workers have a negative impact on the left-behind children's bad behaviors like smoking, drinking, fighting, cyber addiction, and the occurrence rate of such risky behaviors is higher compared with non- left-behind children[3]. Liu and Wang found in 2014 that left-behind boys have higher score in bad communication behavior compared with non-left-behind boys, left-behind girls have higher score in timidity of social communication compared with non-left-behind girls, and that left-behind children's overall social adaptation ability is lower[4]. In cognition, Wang and Chen found in their survey in 2010 that some neutral external information in the daily life is mostly interpreted as negative meaning by left-behind children, which includes not only the negation of themselves but also cognitive errors towards others. They are remarkably different from the non-left-behind children in this regard [5].

The research of rural children's behavior status mostly focuses on rural left-behind children, but there are few researches on the behavior of rural children as a whole. The research method is mainly comparing the behaviors of left-behind children with those of non-left-behind children. The research dimensions focus on the disparity in learning, life and cognition, and the research results usually show rural children's deviant behavior. Rural left-behind children are more likely to have behavioral deviation or other problems due to inadequate supervision, but rural non-left-behind children also need social attention and help. Seen from current literatures, the problems of left-behind children have got more attention, and many studies demonstrate the stale concept that deviant behavior results from lack of parentage, which is not conducive to the healthy growth of rural left-behind children and even produces negative impact. "Problem child" has become the label of left-behind children. This study investigated the behavior status of rural children through a survey, drew conclusions based on factor analysis, and put forward corresponding suggestions to promote the healthy development of rural children's behavior. 


\section{RESEARCH METHODS}

In this study, a quantitative method was used to complete the survey from January 2016 to July 2016, and the data were collected and analyzed by STATA13.0.

In order to ensure the Representative of the samples, and improve the measurement reliability and validity, this investigation adopts random sampling method in the central region of China - Zhushan County, Shiyan City, Hubei province. Thirty seven primary and secondary schools in 17 towns were randomly selected, and 5,000 questionnaires were handed to students from the 1 st grade to the 9 th grade. Finally 4,889 copies were returned, accounting for $97.8 \%$ of all copies.

According to the 29 variables related to behavior, 400 samples with missing values were eliminated, and there are 4,489 effective samples. Factor analysis was used to analyze the behavioral status variables of rural children. Based on the study of the internal dependence among the variables, the multiple measured variables are transformed into a few irrelevant aggregate indicators (i.e. factors), and the dimension reduction is achieved by simplifying the data.

\section{RESULTS}

\section{A. Exploratory Factor Analysis of Rural Children's Behavior Status}

\section{1) Data Test of KMO Value}

The data were tested and analyzed, and the KMO value test showed that the correlation between variables and the KMO test value is 0.7095 . The KMO value is greater than 0.7 , indicating that factor analysis can be done, so this set of data is suitable for factor analysis.

\section{2) Factor Analysis}

Through the observation of the scree plot (Fig. 1), it was found that the data after the third factor showed a relatively gentle trend, so it was decided to select the first 3 factors.

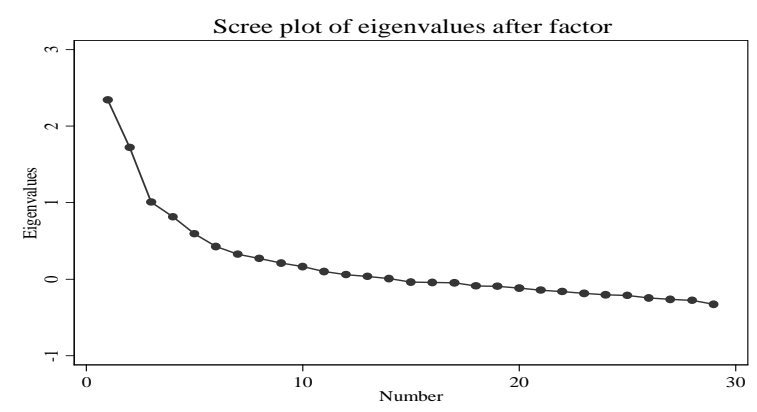

Fig. 1. Scree plot of eigenvalues after factor

TABLE I. EIGENVALUE, VARIANCE CONTRIBUTION RATE AND CUMULATIVE VARIANCE CONTRIBUTION RATE OF COMMON FACTORS

\begin{tabular}{cccc}
\hline $\begin{array}{c}\text { Common } \\
\text { Factor }\end{array}$ & Eigenvalue & $\begin{array}{c}\text { Variance } \\
\text { Contribution } \\
\text { Rate \% }\end{array}$ & $\begin{array}{c}\text { Cumulative } \\
\text { Variance } \\
\text { Contribution } \\
\text { Rate \% }\end{array}$ \\
\hline Factor1 & 2.06434 & 36.77 & 36.77 \\
Factor2 & 1.59508 & 28.41 & 65.17 \\
Factor3 & 1.40722 & 25.06 & 90.24 \\
\hline
\end{tabular}

The calculation results of common factor eigenvalues show that there are three factors whose eigenvalue is bigger than 1, therefore, the three factors are retained. The explainable cumulative variance contribution rate is $90.24 \%$, the unexplained information of variable is $9.76 \%$, and the population variance's variable information explanation ability is relatively good. According to table 1 , it can be found that the behavior status of rural children is reflected more prominently in the first common factor, with the variance contribution rate as $36.77 \%$; the other two factors in descending order is F2 (28.41\%) and F3 (25.06\%) according to the contribution rate.

In order to highlight the differences among factors, the maximum variance method is used to rotate and the factor load matrix is obtained. In order to better measure the behavior status of rural children and make the research more scientific and standardized, it is determined that the variables which are not representational and whose load value is less than 0.4 be excluded. Through screening, there are three variables whose load values are greater than 0.4 in the first common factor, and they are respectively v20 (0.5348), v27 (0.5894), v29 (0.5488); there are three variables whose load values are greater than 0.4 in the second common factor, and they are respectively v13 (0.5048), v14 (0.6486), v15 (0.6693); there are two variables whose load values are greater than 0.4 in the third common factor, namely v9 (0.4742) and v12 (0.4892).

In order to understand the correlation between public factors and rural children's behavior status, the scores of the variables of these three factors are calculated, and the average score is calculated. The results are shown in Table 2.

TABLE II. SCORES OF FACTORS

\begin{tabular}{llcc}
\hline \multicolumn{1}{c}{ Variable } & \multicolumn{3}{c}{ Factor } \\
\cline { 2 - 4 } & F1 & F2 & F3 \\
\hline Resort to parents for help when & & & 0.21812 \\
encountering difficulties in life v9 & & 0.22577 \\
Resort to teachers for help when & & \\
encountering difficulties in life v12 & & \\
Resort to the government for help & & \\
when encountering difficulties in life & & & \\
v13 & & 0.19394 & \\
Resort to the school for help when & & \\
encountering difficulties in life v14 & & \\
Resort to social organizations for & & \\
help when encountering difficulties \\
in life v15
\end{tabular}

\section{B. Analysis on Results of Factors}

A total of three factors (including 11 variables) are extracted in exploratory factor analysis. The three factors are named as follows: Factors affecting the behavior status of rural children include communication and learning, seeking help from organizations and seeking help from individuals.

Factor One includes three variables: willingness to make a compromise when in conflict with classmates or friends v20, what to do when mistakes are made v27, and time to do 
homework after school v29. Through the analysis of these three variables, v20 and v27 reflect how the rural children handle relationships with others, so the two variables are understood as communication behavior; v29 describes the time of doing homework after school by rural children, so it is understood as learning behavior. The three variables reflect the behavior of rural children in communication and learning. And the three variables have relatively high load value, indicating that their correlation with Factor One is relatively high, so the first common factor is named as "communication and learning behavior”.

Factor Two includes three variables: resort to the government for help when encountering difficulties in life v13, resort to the school for help when encountering difficulties in life v14, and resort to social organizations for help when encountering difficulties in life v15. The analysis of these three variables reflects the organizations from which children can seek help. Meanwhile, these three variables have relatively high load value. Therefore, Factor Two is named as "behavior of seeking help from organizations" by integrating the characteristics of the three variables.

Factor Three includes two variables: resort to parents for help when encountering difficulties in life v9 and resort to teachers for help when encountering difficulties in life v12. The analysis found that in the face of difficulties in life, rural children will seek help and support from parents and teachers who are closer to them in their lives. Factor Three and Factor Two both reflect from where rural children seek help when encountering difficulties in life, but the difference is that Factor Three reflects the individuals from whom rural children seek help. The two variables have relatively high load values and their correlation with Factor Three is relatively high. By integrating, Factor Three is named as "behavior of seeking help from individuals".

\section{DISCUSSION}

\section{A. Factor of the Behavior of Seeking Help from Organizations}

The scores of the three variables in this factor are 0.19394 points (v13), 0.30899 points (v14) and 0.34767 points (v15). The average score of the three variables is 0.28353 points. By comparing the scores of variables, it can be found that the relationship between resorting to social organizations for help (v15) and the factor of the behavior of seeking help from organizations is the most significant when facing difficulties in life.

Because of age characteristics, living environment and other factors, rural children will encounter difficulties from life, learning, emotion and other aspects. Seeking help is one of the methods used to get out the plight. This strategy contains the troubled children's self-perception of difficulties, selection of helpers and methods of seeking help as well as assessment of results of help-seeking. The behavior of seeking help is directly related to the social development of children.

According to the results of factor analysis, the factor of the behavior of seeking help from organizations is found to be the most important factor affecting the behavior of rural children. Among them, resorting to social organizations for help is the most important variable affecting the behavior of rural children. At present, China's rural social organizations have begun to take shape, and play an important role in helping rural children. However, there are still many problems in social organizations, such as external management and self-development, which seriously restrict their function and sustainable development. In order to better give play to the role of social organizations in helping left-behind children, relevant legal system of social organizations should be improved, and the nature, purpose, organizational structure, internal system, sources of funds and civil legal relationship of social organizations should be clarified in the form of law[6]. Secondly, it is advised to accelerate the construction of service-oriented government, lower the threshold in the registration and management of social organizations and simplify procedures. Meanwhile, part of public services should be transferred to social organizations, and guidance and financial support for rural social organizations should be strengthened. Finally, social organizations themselves should constantly improve the internal structure, and strive to broaden the sources of funds, strengthen staffing, and improve the professional quality of their members.

\section{B. Factor of Communication and Learning Behavior}

The three variables in this factor are 0.19078 points (v20), 0.23465 points (v27) and 0.23372 points (v29), and the average level of the three variables is 0.21972 points.

Communication and learning behavior is the second important factor affecting the behavior of rural children. Childhood is an important period for the development of human behaviors, and the social intercourse in this period has a great influence on the formation of children's personality [7]. Good interpersonal communication has positive effects on children's psychological health, self-cognition and information exchange.

Through the factor analysis, it is found that rural children are more willing to make a compromise when in conflict with classmates or friends, which reflects their positive relationship with others and their intent for good communication performance. In order to promote the ability of rural children to communicate in practice, professional social work can be carried out. First, it is about dealing with casework. On the one hand, it is advised to build relationship with rural children so as to gradually carry out work; on the other hand, specific work should be done based on the actual situation of the family and it is better to communicate with the parents. Second, it is about group work. Various group activities should be regularly organized to enhance communication between members, encourage members of the group to change themselves, and to promote members' interpersonal skills.

On the other hand, the variable of "time to homework after school” reflects the attitude of rural children towards learning. $\mathrm{Li}$ argued based on his survey in 2012 that academic achievement is an important indicator to measure children's academic adaptation, and that learning attitude and learning achievement is positively related [8].

In order to better promote the rural children's academic adjustment and improve their academic achievement, efforts 
can be made in the following aspects. On the family side, the guardians should realize the importance of family education, and give their children academic care and encouragement as much as possible. They also should keep in touch with the school, and actively understand about the current situation of children's learning. On the school side, the school's management division should construct the mechanism of school development and construction, and optimize the educational environment of rural schools. Teachers should help stimulate rural children's interest in learning, improve their learning motivation, and develop good learning habits. And they should also guide rural children to do self-education and self-management so as to enhance their independence. As for the government, it should increase investment in rural schools and gradually narrow the gap between rural areas and urban areas in terms of funding, school conditions, faculty, teaching level and other aspects in a bid to improve the quality of education in rural areas.

\section{Factor of the Behavior of Seeking Help from Individuals}

The score of the two variables is 0.21812 points (v9) and 0.22577 (v12), and their average score is 0.221945. The behavior of seeking help from individuals is the third important factor affecting the behavior of rural children.

Chu, Hsieh and other researchers found in 2011 that juveniles tend to turn to their parents and peers for help[9]. However, their parents have not received professional training in dealing with children's emotional and personal problems, which makes the help-seeking limited in effectiveness. At the same time, Rickwood and other researchers in 2007 pointed out those parents are the first choice for children to seek help, and that they play an important role in the transfer from seeking non-professional psychological help and seeking professional psychological help [10]. Therefore, it is advised to enhance the effectiveness of children's parental help through providing parenting education for parents of rural children. Parenting education can not only promote parents' education concept and communication skills, but also creates a more optimized family ecological environment for the healthy growth of rural children.

At present, the parenting education in China's rural areas can be boosted from the following aspects: On the side of government, it should formulate corresponding laws and regulations on parenting education and specify the content and measures of parenting education. At the same time, a special guidance institution should be set up to help parents improve their cognitive level of parenting education in rural areas. On the side of school, primary and secondary schools in rural areas should play the role of main channel in parenting education. They can join professional organizations to carry out various parenting education activities according to the age and family situation of rural children so that their parents can acquire more methods and skills of parent-child communication under professional guidance. As for parents, they can improve their knowledge level through the Internet, television, lectures and other ways, constantly broaden knowledge, understand and grasp the concept of modern education, promote communication with their children, thus providing good family support for rural children's healthy growth.
The education level of teachers is generally higher than that of rural children's parents. The teachers have more communication skills and are more able to understand children's learning and life. Therefore, they can actively guide children to take mature and rational ways when facing difficulties and can effectively help rural children out of problems. However, due to the living environment, salary and other reasons, the overall quality of rural teachers is generally lower than that of urban areas. Therefore, various measures should be adopted to enhance the professional quality of rural teachers so that they can better respond to rural children's help-seeking behavior and promote children's healthy growth.

\section{SUMMARY}

Factor of the behavior of seeking help from organizations is found to be the most important factor affecting the behavior of rural children. Social organizations play an important role in helping rural children. The state has established and perfected relevant laws. The government has increased its support for all aspects of social organizations and the social organizations themselves have stepped up their efforts. Communication and learning behavior is the second important factor affecting the behavior of rural children. The rural children in the survey showed positive performance in interpersonal communication and learning. Through case work and group work, children can be helped to enhance their interpersonal skills, and help children to improve their academic adaptability through family, school and government. The behavior of seeking help from individuals is the third important factor affecting the behavior of rural children. Through the parental education of parents and improve the quality of teachers in rural areas to enhance the effectiveness of children seeking help.

\section{REFERENCES}

[1] Y. C. Tang, P. Fu. The Effect of Parent-Child Separation on Left-Behind Children - Based on Empirical Research of Specific Parent-Child Separation [J]. Population Journal, 2011 (5): 41-49.(In Chinese)

[2] C. J. Ji. Study on Education Plight of Left-Behind Children from Difference in Academic Achievement -- Based on Empirical Investigation of Left-Behind and Non -Left-Behind Children [J]. Shanghai Research on Education, 2016 (4): 41-46.(In Chinese)

[3] G. Y. Li , T. M. Luo , F. B. Tao . The Impact of Parents as Migrant Workers on Left-behind Children's Risky Behaviors and Psychological Factors [J]. Chinese Journal of Public Health, 2012, 28 (7): 924-926. (In Chinese)

[4] T. Liu , Y. R. Wang , L. Q. Xu , et al. Investigation and Research on Behavior Problems of Left-behind Children and Non-left-behind Children in Rural Areas of Lichuan [J]. Maternal and Child Health Care of China, 2015, 30 (35): 6307-6308. (In Chinese)

[5] X. D. Wang, X. Chen. Comparative Study on Social Anxiety and Cognitive Bias of Left-behind Children and Non-left-behind Children [J]. Journal of Sichuan Normal University (Social Sciences Edition), 2010, 37 (2): 57-61. (In Chinese)

[6] Y. B. Zhu. Research on the Development of Rural Non-Profit Organizations from the Perspective of New Socialist Countryside Construction. Rural Economy. 2013 (3): 44-50. (In Chinese)

[7] Philip G. Zimbardo, Robert Johnson, Vivian McCann. Psychology: Core Concepts (8th Edition) .Pearson.2016.

[8] B. H. Li. Study on Rural Junior Middle School Students' Learning Motivation, Learning Attitude and Academic Achievement [J]. Journal of Hunan University of Science and Technology (Social Sciences Edition), 2012, 15 (4): 146-149. (In Chinese) 
[9] Chu J P, Hsieh K Y, Tokars D A. Help-seeking tendencies in Asian Americans with suicidal ideation and attempts.[J]. Asian American Journal of Psychology, 2011, 2(1):25-38.

[10] DJ Rickwood, FP Deane, CJ Wilson. When and how do young people seek professional help for mental health problems?[J].The Medical Journal of Australia.2007(187):35-39. 